\title{
Hopf Algebras of Combinatorial Structures ${ }^{1}$
}

\author{
William R. SchmitT \\ Department of Mathematical Sciences \\ Memphis State University \\ Memphis, Tennessee 38152
}

\begin{abstract}
A generalization of the definition of combinatorial species is given by considering functors whose domains are categories of finite sets, with various classes of relations as morphisms. Two cases in particular correspond to species for which one has notions of restriction and quotient of structures. Coalgebras and/or Hopf algebras can be associated to such species, the duals of which provide an algebraic framework for studying invariants of structures.
\end{abstract}

${ }^{1} 1980$ Mathematics Subject Classification (1985 Revision). Primary 16A24; Secondary 05A99, 18B99. 


\section{INTRODUCTION}

The theory of species, first developed by André Joyal in 1981 (see [5]), has provided a pliable language for combinatorial enumeration which has since been extensively used (see e.g. [2], [6], [7], [8], [9], [10]). The purpose of the present work is twofold. First, Joyal's notion of species is extended in such a way that combinatorial structures are classified according to their functorial properties. In particular, the existence of substructures and quotient structures can be expressed in much the same way that the notion of species renders the concept of a combinatorial structure generally. We do this by a slight change in the definition of a species. Instead of taking the category of finite sets and bijections as our starting point, as Joyal does, we consider some notable subcategories of the category of finite sets and all relations. Of particular importance are the categories having partially defined bijections and partially defined surjections as morphisms. Functors from these two categories to the category of sets provide the definition of species for which "restriction" and "quotient" of structures are defined, respectively.

Second, we reexamine the objective of Joyal's machinery from the point of view of Hopf algebras. Joyal's notion of species is, roughly speaking, a set-theoretic analog of the concept of generating function, and algebraic properties of generating functions find pleasing - and, we believe, definitive - equivalent renderings in species-theoretic terms. Thus, in theory, one might altogether dispense with the use of generating functions in enumeration, and deal directly with the objects themselves. Such an approach, however, puts greater focus than ever on the problem of determining the "natural" algebraic operations to be used in studying a given family of combinatorial structures, operations which should be dictated by the structures themselves, rather than via numerical devices, such as generating functions.

We appoach this problem from the point of view of the theory of Hopf algebras. It has long been noticed that various decompositions of combinatorial objects can be most clearly expressed using the concept of coproduct in a coalgebra. When a suitable product of objects under consideration is defined, usually corresponding to disjoint union, the associated coalgebra inherits a bialgebra, or Hopf algebra, structure. The question thus arises as to what is the minimum of additional structure that is to be imposed on a species, in order that a coalgebra and/or Hopf algebra can be naturally associated.

Remarkably, it turns out that the generalizations we propose in order to speak the language of "substructure" and "quotient structure" are just such minimal requirements. The Hopf algebras thus obtained are generalizations of two classical examples of Hopf algebras, namely, the Hopf algebra of polynomials in one variable, and the Faà di Bruno Hopf algebra (see [4]). We thereby obtain a large variety of new examples of Hopf algebras. Furthermore, the correspondences established between species, on one hand, and coalgebras and Hopf algebras, on the other, are functorial. 
The Hopf algebras associated with species may be viewed as sophisticated counting schemes which supplement, or perhaps even replace, the naive use of generating functions in enumeration.

\section{Relational Categories and Species}

A relation $f: U \rightarrow V$ from a set $U$ to a set $V$ is a subset of the cartesian product $U \times V$. The domain and the range of $f: U \rightarrow V$ are the sets $D(f)=\{x \in U:(x, y) \in f$, for some $y \in V\}$ and $R(f)=\{y \in V:$ $(x, y) \in f$, for some $x \in U\}$, respectively. If $f: U \rightarrow V$ and $g: V \rightarrow W$ are relations, then the composition $g \circ f: U \rightarrow W$ is the set $\{(u, w):(u, v) \in$ $f$ and $(v, w) \in g$, for some $v \in V\}$. The converse of a relation $f: U \rightarrow V$ is the relation $f^{-1}: V \rightarrow U$ given by $f^{-1}=\{(v, u):(u, v) \in f\}$.

Let Rel denote the category having all finite sets as objects and all relations as morphisms. A relational category is a subcategory of Rel which contains all bijections in its class of morphisms. Since all relational categories have the same class of objects, we refer to them just by naming their morphisms.

The set of all relational categories forms a partially ordered set $\mathcal{C}$, ordered by inclusion of morphism classes. This partially ordered set is actually a lattice, for if $\mathbf{C}_{1}$ and $\mathbf{C}_{2}$ are relational categories, then their least upper bound, or join, $\mathbf{C}_{1} \vee \mathbf{C}_{2}$ is generated by taking all compositions in Rel of morphisms from the categories $\mathbf{C}_{1}$ and $\mathbf{C}_{2}$, and the greatest lower bound, or meet, $\mathbf{C}_{1} \wedge \mathbf{C}_{2}$ is obtained by intersecting the classes of morphisms of $\mathbf{C}_{1}$ and $\mathbf{C}_{2}$. The maximal element of $\mathcal{C}$ is $\mathbf{R e l}$, and the minimal element is the category $\mathbf{B}$ of bijections.

The following relational categories are of basic importance: I, injections; $\mathbf{I}^{o p}$, coinjections (converses of injections); $\mathbf{S}$, surjections; and $\mathbf{S}^{o p}$, cosurjections (converses of surjections). In fact, we have the following result.

Proposition 2.1. The category $\mathbf{R e l}$ is equal to the join $\mathbf{I} \vee \mathbf{I}^{o p} \vee \mathbf{S} \vee \mathbf{S}^{o p}$ in the lattice $\mathcal{C}$ of all relational categories.

Proof. Let $f: U \rightarrow V$ be any morphism in Rel. Define $f_{1}: U \rightarrow D(f)$ to be the coinjection $\{(x, x): x \in D(f)\}$, and let $f_{2}: D(f) \rightarrow f$ be the cosurjection $\{(x,(x, y)):(x, y) \in f\}$. Let $f_{3}: f \rightarrow R(f)$ be the surjection $\{((x, y), y)$ : $(x, y) \in f\}$, and define $f_{4}: R(f) \rightarrow V$ to be the injection $\{(y, y): y \in R(f)\}$; then the relation $f$ is equal to the composition $f_{4} \circ f_{3} \circ f_{2} \circ f_{1}$.

The various joins of the categories $\mathbf{I}, \mathbf{I}^{o p}, \mathbf{S}$, and $\mathbf{S}^{o p}$ are fundamental. For example, the category $\mathbf{F}$ of functions is equal to $\mathbf{S} \vee \mathbf{I}$, because any function factors as an injection composed with a surjection. The join $\mathbf{S} \vee \mathbf{I}^{o p}$ is the category $\mathbf{S}_{p}$ of partially defined surjections. A morphism $f: U \rightarrow V$ in $\mathbf{S}_{p}$ consists of a pair $(W, \bar{f})$, where $W \subseteq U$, and $\bar{f}$ is a surjection from $W$ onto $V$. We also have the categories $\mathbf{F}_{p}=\mathbf{I} \vee \mathbf{I}^{o p} \vee \mathbf{S}$, of partially defined functions, and $\mathbf{I}_{p}=\mathbf{I} \vee \mathbf{I}^{o p}$, of partially defined injections. 
There are many other relational categories besides $\mathbf{B}$ and the joins that can be formed from the set $\left\{\mathbf{I}, \mathbf{I}^{o p}, \mathbf{S}, \mathbf{S}^{o p}\right\}$. For example, if $k$ is some fixed positive integer, one has the relational category of all surjections $f$ such that each block of the kernel of $f$ has $k^{r}$ elements, for some $r \geq 0$ which depends on the choice of $f$. It should be interesting to classify all relational categories, and thus attempt to determine the structure of the lattice $\mathcal{C}$.

Definition 2.2. A species is a functor from some relational category to the category $\mathbf{F}$ of functions.

Usually it is important to specify on which category a species is defined. Thus a species having a relational category $\mathbf{C}$ as its domain is called a C-species.

The above is a refinement of the definition of species given in [5], as a functor from the category of bijections to itself. Such species can be identified with $\mathbf{B}$-species in the obvious manner.

Suppose $\mathbf{C}$ is a relational category and $F$ is a $\mathbf{C}$-species. An F-structure on a set $V$ is an ordered pair $(G, V)$, where $G \in F(V)$. In general, we assume the underlying set $V$ is understood and refer to the elements of $F(V)$ themselves as $F$-structures on $V$. If $G \in F(V), H \in F(U)$, and $H=F[\varphi](G)$ for some bijection $\varphi: V \rightarrow U$, then $G$ and $H$ are said to be isomorphic, denoted by $G \simeq H$. Isomorphism is an equivalence relation on the class of all $F$-structures. The equivalence class containing an $F$-structure $G$, denoted by $[G]$, is called the isomorphism class or the type of $G$.

Two $F$-structures $G$ and $H$ are weakly isomorphic, denoted $G \sim H$, if there is a relation $\alpha: V \rightarrow U$ in $\mathbf{C}$ with converse $\alpha^{-1}$ also in $\mathbf{C}$, such that $F[\alpha](G)=H$ and $F\left[\alpha^{-1}\right](H)=G$. Weak isomorphism is also an equivalence relation on $F$-structures. The weak isomorphism class of an $F$-structure $G$ will be denoted by $\langle G\rangle$. For any species $F$, the collections $\tilde{F}$ of all isomorphism classes and $\hat{F}$ of all weak isomorphism classes of $F$ structures form countably infinite or, in some cases, finite sets.

For any relational category $\mathbf{C}$, the category of $\mathbf{C}$-species is the category having all $\mathbf{C}$-species as objects and natural transformations as morphisms. Thus a morphism $\alpha: F \rightarrow E$ between $\mathbf{C}$-species $F$ and $E$ consists of maps $\alpha_{V}: F(V) \rightarrow E(V)$ for all finite sets $V$, such that whenever $g: V \rightarrow U$ is a relation in $\mathbf{C}$, the diagram

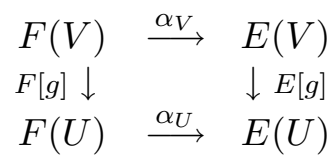

commutes. If $\alpha_{V}$ is a bijection for all $V$, then $\alpha: F \rightarrow E$ is an isomorphism of species. We usually write $F=E$, if $F$ and $E$ are isomorphic species.

\section{Species With Restrictions}

3.1. Definition and Examples. Let $\mathbf{I}^{o p}$ be the category of coinjections. A species with restrictions, or $R$-species, for short, is an $\mathbf{I}^{o p}$-species $F$ which 
satisfies $|F(\emptyset)|=1$. Let $V$ be a finite set, $U$ a non-empty subset of $V$, and let $\rho_{V, U}: V \rightarrow U$ be the converse of the inclusion map from $U$ into $V$. The relation $\rho_{V, U}$ is a coinjection, called the coinclusion from $V$ to $U$. Suppose $F$ is an R-species and $G \in F(V)$ is an $F$-structure on V. The $F$-structure $F\left[\rho_{V, U}\right](G)$ on $U$ is called the restriction of $G$ to $U$, and denoted by $G \mid U$. If $U$ is the empty-set, then $G \mid U$ is equal to the one-element set $F(\emptyset)$. If $W$ is a subset of $U$, then the functoriality of $F$ together with the fact that $\rho_{V, W}=\rho_{U, W} \circ \rho_{V, U}$ implies that

$$
(G \mid U)|W=G| W .
$$

Example 3.1 (R-Species).

1) The uniform species $U$ is defined by $U(V)=\{V\}$ for all sets $V$. Since there is only one $U$-structure on any set, restrictions can be defined in only one way, thus $U$ is an R-species.

2) A graph is simple if it has no loops or multiple edges. Thus, a simple graph can be defined as a pair $(V, E)$, where $V$ is a finite set and $E$ is a set of pairs of elements of $V$. Let $G_{s}(V)$ be the set of all simple graphs with vertex-set $V$. If $H \in G_{s}(V)$ and $U \subseteq V$, let $H \mid U$ be the induced subgraph of $H$, with vertex-set $U$ and edge-set consisting of all edges of $H$ which are contained in $U$. Thus $G_{s}$ is an R-species.

3) Let $M(V)$ denote the set of all matroids having point set $V$. $M$ is an R-species in two different ways: Given $N \in M(V)$ and $U \subseteq V$, one can define $N \mid U$ as either the matroid restriction or contraction of $M$ to $U$.

4) Let $L(V)$ be the set of all linear orderings of the set $V$. Given a linear order on $V$, any subset of $V$ naturally inherits a linear ordering. Thus $L$ is an R-species.

$5)$ Let $C(V)$ denote the set of all cyclic orderings of the set $V$. Any cyclic ordering of $V$ naturally induces cyclic orderings on all subsets of $V$. Thus $C$ is an R-species.

6) Let $S(V)$ be the set of all simplicial complexes on the set $V$. If $G \in S(V)$ and $U \subseteq V$, then $G \mid U$ is the simplicial complex $\{W \cap U: W \in G\}=\{W \in$ $G: W \subseteq U\}$.

7) Let $\mathcal{G}$ be any family of graphs which is closed under the formation of vertex-induced subgraphs, and let $F_{\mathcal{G}}(V)$ be the set of all graphs with vertexset $V$ which are isomorphic to some element of $\mathcal{G}$. Then $F_{\mathcal{G}}$ is an R-species. Some examples are: forests; planar graphs; complete graphs; all $k$-colorable graphs, for some fixed $k$; all graphs having some fixed excluded set of minors; and all vertex-induced subgraphs of some fixed graph $H$.

8) Let $F_{r}(V)$ be the set of all forests $G$ having vertex-set $V$, such that each tree in $G$ has a distinguished "root" vertex. If $G$ is a forest on $V$ and $U \subseteq V$ then the induced subgraph $G \mid U$ is clearly a forest. For each tree $T$ in $G \mid U$, let $T^{\prime}$ be the tree in $G$ having $T$ as a subtree. Define the root of $T$ to be the (unique) vertex of $T$ which is closest (in $T^{\prime}$ ) to the root of $T^{\prime}$. Hence $F_{r}$ is an R-species.

Example 3.2 (Morphisms of R-Species). 
1) For any R-species $F$ with $F(V) \neq \emptyset$, for all $V$, there is a natural transformation $\mu$, from $F$ to the uniform species $U$, where $\mu_{V}$ is the unique map from $F(V)$ to the one-element set $U(V)$, for each set $V$.

2 ) Given any linear order on the set $V$ one can construct a cyclic order on $V$ by letting the minimal element of $V$ be the immediate successor of the maximal element of $V$. This defines a morphism of R-species $L \rightarrow C$.

3) A morphism $\chi: G_{s} \rightarrow S$ from the R-species of graphs to the R-species of simplicial complexes is given by letting $\chi_{V}(G)$ be the collection of independent subsets of the vertex set $V$ of the graph $G$.

4) Let $M_{r}$ and $M_{c}$ be the R-species of matroids with restriction to subsets given by matroid restriction and contraction respectively. For any set $V$, define $d_{V}: M_{r}(V) \rightarrow M_{c}(V)$ by $d_{V}(N)=N^{*}$, the dual matroid of $N$. The maps $d_{V}$ define a isomorphism of R-species $d: M_{r} \rightarrow M_{c}$.

3.2. Cocommutative Coalgebras of R-Species. From now on, $K$ will be some fixed commutative ring with identity. Given an R-Species $F$, let $\mathcal{B}_{F}$ be the free $K$-module having the set $\tilde{F}$ of isomorphism types of $F$-structures as a basis. Define linear maps $\Delta: \mathcal{B}_{F} \rightarrow \mathcal{B}_{F} \otimes \mathcal{B}_{F}$ and $\epsilon: \mathcal{B}_{F} \rightarrow K$ by

$$
\Delta[G]=\sum_{U \subseteq V}[G \mid U] \otimes[G \mid V-U],
$$

and

$$
\epsilon[G]= \begin{cases}1 & \text { if } V=\emptyset \\ 0 & \text { otherwise }\end{cases}
$$

for any $F$-structure $G$ on a set $V$.

Proposition 3.1. For any $R$-species $F$, the $K$-module $\mathcal{B}_{F}$ is a cocommutative $K$-coalgebra, with comultiplication $\Delta$ and counit $\epsilon$ defined as above.

Proof. First we need to show that $\Delta$ is well-defined by 3.2. In order to do this, suppose that $[G]=[H]$ for some $G \in F(V)$ and $H \in F(W)$. Then there is a bijection $\varphi: V \rightarrow W$ such that $F[\varphi](G)=H$. Using the bijection $\varphi$, we can write $\Delta[H]$ as

$$
\Delta[H]=\sum_{U \subseteq V}[H \mid \varphi(U)] \otimes[H \mid \varphi(V-U)] .
$$

It follows from the functoriality of $F$ that $G|U \simeq H| \varphi(U)$ for any subset $U$ of $V$; thus, comparing the above expression for $\Delta[H]$ with equation 3.2 , we see that $\Delta[G]=\Delta[H]$, and so $\Delta$ is well-defined. If $\Delta$ is applied either to all of the terms on the left, or all of the terms on the right side of the tensor product in equation 3.2, then in either case we obtain (again, using the functoriality of $F$ ) the sum

$$
\sum_{U_{1}, U_{2}, U_{3}}\left[G \mid U_{1}\right] \otimes\left[G \mid U_{2}\right] \otimes\left[G \mid U_{3}\right],
$$

taken over all all ordered triples $U_{1}, U_{2}, U_{3}$ of pairwise disjoint subsets of $V$ whose union is equal to $V$. Thus $\Delta$ is coassociative. It is easy to see that $\epsilon$ 
is well-defined and has the counitary property

$$
\sum_{U \subseteq V} \epsilon[G \mid U] \cdot[G \mid V-U]=\sum_{U \subseteq V}[G \mid U] \cdot \epsilon[G \mid V-U]=[G] .
$$

Thus $\mathcal{B}_{F}$ is a coalgebra. The cocommutativity of $\mathcal{B}_{F}$ is obvious.

Suppose $F$ and $E$ are R-species and $\alpha: F \rightarrow E$ is a natural transformation. Then there is a linear map $\tilde{\alpha}: \mathcal{B}_{F} \rightarrow \mathcal{B}_{E}$ defined by

$$
\tilde{\alpha}[G]=\left[\alpha_{V}(G)\right],
$$

for any $F$-structure $G$ on a set $V$.

Proposition 3.2. If $\alpha: F \rightarrow E$ is a morphism of $R$-species, then the corresponding map $\tilde{\alpha}: \mathcal{B}_{F} \rightarrow \mathcal{B}_{E}$ is a coalgebra map.

Proof. Let $G$ be an $F$ structure on a set $V$. Then

$$
\Delta \circ \tilde{\alpha}[G]=\sum_{U \subseteq V}\left[\alpha_{V}(G) \mid U\right] \otimes\left[\alpha_{V}(G) \mid V-U\right] .
$$

Since $\alpha$ is a natural transformation, this can be written as

$$
\sum_{U \subseteq V}\left[\alpha_{U}(G \mid U)\right] \otimes\left[\alpha_{V-U}(G \mid V-U)\right]
$$

which is equal to $(\tilde{\alpha} \otimes \tilde{\alpha}) \circ \Delta[G]$. Also, $\tilde{\alpha}$ preserves the counit $\epsilon$, because $\epsilon[G]$ depends only on the size of $V$, Therefore $\tilde{\alpha}$ is a coalgebra map.

We thus have the following theorem.

Theorem 3.3. The correspondence $F \mapsto \mathcal{B}_{F}$ is a functor from the category of $R$-species and natural transformations to the category of cocommutative coalgebras and coalgebra maps.

3.3. Cocommutative Hopf Algebras of Exponential R-Species. Suppose $F$ is a species. An assembly of $F$-structures is a finite set of $F$-structures whose underlying sets are nonempty and mutually disjoint. If $F$ satisfies $F(\emptyset)=\emptyset$, then the exponential of $F$ is the $\mathbf{B}$-species $E=\exp F$ whose structures are assemblies of $F$-structures. Thus an $E$-structure $G$ on a set $V$ has the form $G=\left\{G_{B}: B \in \pi_{G}\right\}$, where $\pi_{G}$ is a partition of $V$ and $G_{B} \in F(B)$, for each block $B \in \pi_{G}$. The structures $G_{B}$ are the components, and the partition $\pi_{G}$ is the underlying partition, of the $E$-structure $G$. The unique $E$-structure on the empty-set is the empty assembly $\emptyset$. The sum $G+H$ of two assemblies of $F$-structures $G$ and $H$ is the disjoint union of $G$ and $H$. Any assembly $G=\left\{G_{B}: B \in \pi_{G}\right\}$ can thus be written as $G=\sum_{B \in \pi_{G}}\left\{G_{B}\right\}$

If $F$ is any species then the species of non-empty F-structures is the Bspecies $F_{0}$ defined by

$$
F_{0}(V)=\left\{\begin{array}{cc}
F(V) & \text { if } V \neq \emptyset \\
\emptyset & \text { if } V=\emptyset \\
7 &
\end{array}\right.
$$


If $F$ happens to be an R-species, then the exponential $E=\exp F_{0}$ becomes an R-species as follows: if $G=\left\{G_{B}: B \in \pi_{G}\right\}$ is an $E$-structure on $V$ and $U \subseteq V$, the restriction of $G$ to $U$ is defined by

$$
G \mid U=\left\{G_{B} \mid B \cap U: B \in \pi_{G} \text { and } B \cap U \neq \emptyset\right\} .
$$

In other words, the restriction $G \mid U$ of $G$ is obtained by taking the assembly of restrictions of the individual components of $G$.

Any R-species $E$ which coincides with an exponential $\exp F$ on the subcategory $\mathbf{B}$ is called an exponential $R$-species, and in this case we write $E=\exp F$.

Example 3.3 (Exponential R-Species).

1 ) The species of partitions $\Pi$ is equal to the $\exp U_{0}$, where $U$ is the uniform species. Since $U$ is an R-species, it follows that $\Pi$ is also an R-species.

2) The species of permutations $\Sigma$ is equal to $\exp C_{0}$, where $C$ is the species of cyclic orders. Since $C$ is an R-species, it follows that $\Sigma$ is also an R-species. 3 ) The species $G_{s}$ of simple graphs is equal to $\exp G_{c}$, where $G_{c}$ denotes the species of non-empty connected simple graphs. We have already seen that $G_{s}$ is an R-species. The situation here is different from the previous examples, for even if $\left|G_{c}(\emptyset)\right|$ were equal to one, $G_{c}$ would not be an R-species.

Suppose $E=\exp F$ is an exponential R-species. $E$ is coherent if, for any $E$-structure $G=\left\{G_{B}: B \in \pi_{G}\right\}$ on a set $V$, the following condition holds:

$$
G\left|U=\sum_{\substack{B \in \pi_{G} \\ B \cap U \neq \emptyset}}\left\{G_{B}\right\}\right| B \cap U,
$$

for all subsets $U$ of $V$. In particular, if $F$ is an R-species, then $E=\exp F_{0}$ is a coherent $\mathrm{R}$-species whenever restriction of $E$-structures is defined by equation 3.3. The species of simple graphs $G_{s}$ is an example of a coherent exponential R-species which is not of this form.

If $E=\exp F$ is an exponential species, then the set of types $\tilde{E}$ is a commutative monoid, with product given by

$$
[G][H]=[G+H],
$$

for assemblies $G$ and $H$. The identity element of $\tilde{E}$ is $[\emptyset]$, the type of the empty assembly.

If $E=\exp F$ is an R-species, then the coalgebra $\mathcal{B}_{E}$ is also an algebra; that is, the monoid algebra of $\tilde{E}$. The mapping $[G] \rightarrow[\{G\}]$, for all $F$ structures $G$, defines an algebra isomorphism from the polynomial algebra $K[\tilde{F}]$, having types of $F$-structures as indeterminants, onto $\mathcal{B}_{E}$.

Proposition 3.4. Suppose $E=\exp F$ is a coherent, exponential R-species. Then $\mathcal{B}_{E} \simeq K[\tilde{F}]$ is a commutative, cocommutative $K$-Hopf algebra, with 
antipode $S: \mathcal{B}_{E} \rightarrow \mathcal{B}_{E}$ given by

$$
S[G]=\sum_{k=0}^{|V|} \sum_{\substack{\emptyset=U_{0} \subseteq \ldots \subseteq U_{k}=V \\ U_{i} \neq U_{i+1}}}(-1)^{k}\left[G \mid U_{1}-U_{0}\right]\left[G \mid U_{2}-U_{1}\right] \cdots\left[G \mid U_{k}-U_{k-1}\right],
$$

for any E-structure $G$ on a set $V$.

Proof. First, we must show that $\mathcal{B}_{E}$ is a bialgebra, i.e. that $\Delta: \mathcal{B}_{E} \rightarrow$ $\mathcal{B}_{E} \otimes \mathcal{B}_{E}$ and $\epsilon: \mathcal{B}_{E} \rightarrow K$ are algebra maps. If $G_{1} \in E\left(V_{1}\right)$ and $G_{2} \in E\left(V_{2}\right)$ and $V_{1}$ and $V_{2}$ are disjoint, then

$$
\Delta\left(\left[G_{1}\right]\left[G_{2}\right]\right)=\sum_{U \subseteq V_{1} \cup V_{2}}\left[G_{1}+G_{2} \mid U\right] \otimes\left[G_{1}+G_{2} \mid\left(V_{1} \cup V_{2}\right)-U\right],
$$

which is equal to

$$
\sum_{U_{1} \subseteq V_{1}} \sum_{U_{2} \subseteq V_{2}}\left[G_{1}+G_{2} \mid U_{1} \cup U_{2}\right] \otimes\left[G_{1}+G_{2} \mid\left(V_{1} \cup V_{2}\right)-\left(U_{1} \cup U_{2}\right)\right] .
$$

Using the coherence of $E$, equation 3.4, and the definition of product, equation 3.5, this can be written as

$$
\sum_{U_{1} \subseteq V_{1}} \sum_{U_{2} \subseteq V_{2}}\left[G_{1} \mid U_{1}\right]\left[G_{2} \mid U_{2}\right] \otimes\left[G_{1} \mid V_{1}-U_{1}\right]\left[G_{2} \mid V_{2}-U_{2}\right]
$$

which is equal to $\Delta\left[G_{1}\right] \Delta\left[G_{2}\right]$. Hence $\Delta$ is multiplicative. It is trivial to check that $\epsilon$ also is multiplicative.

To see that $\mathcal{B}_{E}$ is a Hopf algebra, we must show that the map $S$ defined by equation 3.6 satisfies the following identity, and is thus an antipode.

$$
\sum_{U \subseteq V} S[G \mid U] \cdot[G \mid V-U]=\sum_{U \subseteq V}[G \mid U] \cdot S[G \mid V-U]= \begin{cases}{[\emptyset]} & \text { if }[G]=[\emptyset] \\ 0 & \text { otherwise }\end{cases}
$$

for any $G \in E(V)$. Equation 3.7 is obvious for $[G]=[\emptyset]$. If $G$ is not empty, the first sum in equation 3.7 can be written as $S[G]$ plus the sum

$$
\sum_{U \subset V} S[G \mid U] \cdot[G \mid V-U]
$$

taken over all subsets $U$ of $V$ with $U \neq V$. Using equation 3.6 for $S$, the sum 3.8 can be written as

$$
\sum_{U \subset V} \sum_{k=0}^{|U|} \sum_{\substack{\emptyset=U_{0} \subseteq \ldots \subseteq U_{k}=U \\ U_{i} \neq U_{i+1}}}(-1)^{k}\left[G \mid U_{1}-U_{0}\right] \cdots\left[G \mid U_{k}-U_{k-1}\right][G \mid V-U],
$$

which equals $-S[G]$, according to formula 3.6. Thus the first sum in equation 3.7 vanishes. The second sum in equation 3.7 vanishes, similarly. 
Note that formula 3.6 for the antipode $S$ of $\mathcal{B}_{E}$ can be written more compactly as

$$
S[G]=\sum_{\pi \in \Pi(V)}(-1)^{|\pi|}|\pi| ! \prod_{B \in \pi}[G \mid B],
$$

where $|\pi|$ denotes the number of blocks of a partition $\pi \in \Pi(V)$.

If $F$ and $E$ are exponential species, a natural transformation $\alpha: F \rightarrow E$ is additive if $\alpha_{U \cup V}(G+H)=\alpha_{U}(G)+\alpha_{V}(H)$, whenever $G$ and $H$ are assemblies of $F$-structures on disjoint sets $U$ and $V$ respectively. If $\alpha$ : $F \rightarrow E$ is additive, where $F=\exp F_{1}$, then it is uniquely defined by its restriction $\alpha_{1}: F_{1} \rightarrow E$. A morphism of exponential species is an additive natural transformation.

If $\alpha: F \rightarrow E$ is a morphism of coherent exponential R-species then the coalgebra map $\tilde{\alpha}: \mathcal{B}_{F} \rightarrow \mathcal{B}_{E}$ is in fact a Hopf algebra map. Thus we have the following theorem.

Theorem 3.5. The correspondence $F \mapsto \mathcal{B}_{F}$ is a functor from the category of coherent exponential $R$-species to the category of commutative, cocommutative Hopf algebras.

Example 3.4 (The Binomial Hopf Algebra). The uniform species $U$ (which is a coherent $\mathrm{R}$-species) is equal to $\exp X$, where $X$ is the singleton species, given by $X(V)=\{V\}$ if $|V|=1$, and $X(V)=\emptyset$, otherwise. Letting $x$ be the unique type of $X$-structure, we see that $\mathcal{B}_{U}$ is isomorphic to the binomial Hopf algebra $K[x]$, where $\Delta x=1 \otimes x+x \otimes 1$, (see [4], [12] ).

Example 3.5 (Partitions). The species of partitions $\Pi=\exp U_{0}$ is a coherent R-species. Let $x_{n}$ be the type of the unique $U$-structure on a set with $n \geq 1$ elements, and let $x_{0}=1$ be the type of the empty partition. The Hopf algebra $\mathcal{B}_{\Pi}$ is isomorphic to the polynomial algebra $K\left[x_{1}, x_{2}, \ldots\right]$, where

$$
\Delta x_{n}=\sum_{k \geq 0}\left(\begin{array}{l}
n \\
k
\end{array}\right) x_{k} \otimes x_{n-k}
$$

for all $n \geq 0$.

If $E=\exp F$ is a coherent R-species, with $F(V) \neq \emptyset$, for all non-empty sets $V$, then the unique morphism $F \rightarrow U$ defines a morphism of exponential species $E \rightarrow \Pi$. Therefore we have a (surjective) Hopf algebra map $\mathcal{B}_{E} \rightarrow$ $\mathcal{B}_{\Pi}$, for all coherent exponential R-species $E$.

3.4. Connected Structures and Lattices of Contractions. Suppose $E=\exp F$ is a coherent R-species. An $E$-structure $G$ is connected if it is an assembly consisting of exactly one $F$-structure. In particular, the empty $E$-structure is not connected, and any $E$-structure on a one element set is connected.

The following proposition justifies the use of the term "connected" in this general context by showing that, when two such structures intersect nontrivially, then their union is connected. This is a basic property of connected objects in any category. 
Proposition 3.6. Let $G \in E(V)$, where $E=\exp F$ is a coherent $R$-species. If $G \mid U_{1}$ and $G \mid U_{2}$ are connected, for $U_{1}, U_{2} \subseteq V$, and $U_{1} \cap U_{2} \neq \emptyset$, then $G \mid U_{1} \cup U_{2}$ is connected.

Proof. Let $\pi_{G \mid U_{1} \cup U_{2}}$ be the underlying partition of the assembly $G \mid U_{1} \cup U_{2}$. By the functoriality of $E, G\left|U_{i}=\left(G \mid U_{1} \cup U_{2}\right)\right| U_{i}$ for $i=1,2$, and by hypothesis, the underlying partition $\pi_{G \mid U_{i}}$ of $G \mid U_{i}$ is equal to $\left\{U_{i}\right\}$, for $i=$ 1,2. By equation 3.4, there must exist blocks $B_{i} \in \pi_{G \mid U_{1} \cup U_{2}}$ such that $U_{i} \subseteq B_{i}, i=1,2$. But then the $B_{i}$ must have non-empty intersection, since $U_{1} \cap U_{2} \neq \emptyset$. This implies that $B_{1}=B_{2}=U_{1} \cup U_{2}$. Thus $G \mid U_{1} \cup U_{2}$ is connected.

If $E=\exp F$ is a coherent R-species and $G$ is an E-structure on a set $V$, a partition $\pi \in \Pi(V)$ is called a contraction of $G$ if $G \mid B$ is connected for all $B \in \pi$. The set of all contractions of $G$, denoted by $\Pi_{c}(G)$, is partially ordered by refinement.

Proposition 3.7. Let $E=\exp F$ be a coherent $R$-species and $G$ be an $E$ structure on a set $V$. The set $\Pi_{c}(G)$ of contractions of $G$ is a sup-sublattice of the partition lattice $\Pi(V)$.

Proof. If $\sigma, \pi \in \Pi_{c}(G)$ then the join $\sigma \vee \pi$ is equal to the join of $\sigma$ and $\pi$ in $\Pi(V)$ because, whenever a block $B$ of $\sigma$ and a block $C$ of $\pi$ have non-empty intersection, $G$ restricted to the union $B \cup C$ is connected by proposition 5 . Let $\tau$ be the meet of $\sigma$ and $\pi$ in $\Pi(V)$. Then in $\Pi_{c}(G), \sigma \wedge \pi=\bigcup_{B \in \tau} \pi_{G \mid B}$, where $\pi_{G \mid B}$ is the underlying partition of the assembly $G \mid B$, for each block $B$ of $\tau$.

$\Pi_{c}(G)$ is thus called the lattice of contractions of $G$. In the case that $E$ is the species $G_{s}$ of simple graphs, $\Pi_{c}(G)$ is the usual lattice of contractions of the graph $G$.

\section{Hereditary Species}

4.1. Definition and Examples. A hereditary species (or H-species, for short) is an $\mathbf{S}_{p}$-species, where $\mathbf{S}_{p}$ is the category of partially defined surjections. Suppose $F$ is an H-species, and $G$ is an $F$-structure on a set $V$. If $\pi \in \Pi(V)$ is a partition of $V$, and $\rho_{V, \pi}: V \rightarrow \pi$ is the canonical surjection, the quotient $G / \pi$ is the $F$-structure on the set $\pi$ defined by $G / \pi=F\left[\rho_{V, \pi}\right](G)$. The restriction $G \mid \pi$ is defined to be the assembly $\{G \mid B: B \in \pi\}$. Now suppose that $\pi$ is a subpartition of $V$, that is, $\pi$ is a partition of some nonempty subset $U$ of $V$. Let $\rho_{V, U}: V \rightarrow U$ be the coinclusion and let $\rho_{U, \pi}: U \rightarrow \pi$ be the canonical surjection. The natural morphism from $V$ to $\pi$ is the partial surjection $\rho_{V, \pi}=\rho_{U, \pi} \circ \rho_{V, U}$. The subquotient $G / \pi$ is the $F$-structure on $\pi$ defined by $G / \pi=F\left[\rho_{V, \pi}\right](G)$.

If $f: V \rightarrow U$ is a partial surjection, then the kernel of $f$, is the subpartition of $V \kappa_{f}=\left\{f^{-1}(x): x \in U\right\}$. So $f$ can be expressed as the composition 
$g \circ \rho_{V, \kappa_{f}}$, where $g: \kappa_{f} \rightarrow U$ is the natural bijection. Therefore, in order to show that a species $F$ is hereditary, it suffices to describe how subquotients of $F$-structures are constructed and to verify functoriality.

Example 4.1 (H-Species).

1) The uniform species $U$ is an H-species.

2 ) The species $L$ of linear orders is an H-species. Suppose $G$ is a linear ordering of a set $V$, and $\pi$ is a subpartition of $V$. The subquotient $G / \pi$ is obtained by ordering the blocks of $\pi$ according to the order of their maximal elements. Of course, this works if we use minimal instead of maximal elements.

3) The species $G_{s}$ of simple graphs is an H-species. Let $G$ be a simple graph with vertex-set $V$, and suppose $\pi$ is a subpartition of $V$. The subquotient $G / \pi$ is defined as the graph with vertex-set $\pi$, having an edge between blocks $B_{1}$ and $B_{2}$ if and only if there is some edge of $G$ which has one endpoint in $B_{1}$ and the other endpoint in $B_{2}$.

4) The species $R$ of relations is an H-species. Suppose $G \in R(V)$ is a relation on $V$. If $h: V \rightarrow U$ is a partial surjection, define $R[h](G): U \rightarrow U$ to be the relation $h \circ G \circ h^{-1}$. If $g: U \rightarrow W$ is another partial surjection then $R[g \circ h](G)=(g \circ h) \circ G \circ(g \circ h)^{-1}=g \circ\left(h \circ G \circ h^{-1}\right) \circ g^{-1}$, which is equal to $R[g] \circ R[h](G)$. Thus $R$ is functorial. Note that we don't use here the fact that the relations $f$ and $g$ are partial surjections. Hence, we have actually shown that the species of relations is a Rel-species, and thus is a $\mathbf{C}$-species for any relational category $\mathbf{C}$.

4.2. Bialgebras of $\mathbf{H}$-species. If $\tau$ and $\sigma$ are partitions of a set $V$ and $\tau \leq \sigma$ (i.e. each block of $\sigma$ is a union of blocks of $\tau$ ), then $\sigma / \tau$ denotes the partition of the set $\tau$ induced by $\sigma$.

Suppose $F$ is an H-species, and $G=\sum_{B \in \pi_{G}}\left\{G_{B}\right\}$ is an assembly of $F$ structures on a set $V$. For $\sigma \leq \pi_{G}$ in $\Pi(V)$ and each block $B$ of $\pi_{G}$, let $\sigma \mid B \in \Pi(B)$ denote the restriction of $\sigma$ to $B$. The restriction of the $G$ to $\sigma$ is the assembly of $F$-structures on $V$ given by $G\left|\sigma=\sum_{B \in \pi_{G}} G_{B}\right|(\sigma \mid B)$, which has $\sigma$ as underlying partition. The quotient of $G$ by $\sigma$ is the assembly on the set $\sigma$ given by $G / \sigma=\sum_{B \in \pi_{G}}\left\{G_{B} /(\sigma \mid B)\right\}$. The underlying partition of $G / \sigma$ is $\pi_{G} / \sigma$.

Proposition 4.1. Suppose $F$ is an $H$-species and $G$ is an assembly of $F$ structures on a set $V$. If $\tau \leq \sigma$ are partitions of $V$, then the following identities hold:

$$
\begin{gathered}
{[(G \mid \sigma) \mid \tau]=[G \mid \tau],} \\
{[(G / \tau) \mid(\sigma / \tau)]=[(G \mid \sigma) / \tau],} \\
{[(G / \tau) /(\sigma / \tau)]=[G / \sigma] .}
\end{gathered}
$$


Proof. It clearly suffices to consider the case that $G$ is an assembly consisting of one element $H \in F(V)$. Suppose $C \in \tau$ and $B \in \sigma$ satisfy $C \subseteq B$. We have from equation 3.1 that $(H \mid B)|C=H| C$. It follows that $(G \mid \sigma) \mid \tau$ and $G \mid \tau$ are identical. So, in particular, they are isomorphic.

If $B \in \sigma$, then the restriction $\tau \mid B$ is an element of the induced partition $\sigma / \tau$. Let $\rho_{V, B}: V \rightarrow B$ and $\rho_{\tau, \tau \mid B}: \tau \rightarrow \tau \mid B$ be the coinclusions and let $\rho_{V, \tau}: V \rightarrow \tau$ and $\rho_{B, \tau \mid B}: B \rightarrow \tau \mid B$ be the canonical surjections. Then $\rho_{B, \tau \mid B} \circ \rho_{V, B}=\rho_{\tau, \tau \mid B} \circ \rho_{V, \tau}$ and hence $(H / \tau) \mid(\tau \mid B)=$ $F\left[\rho_{\tau, \tau \mid B}\right] \circ F\left[\rho_{V, \tau}\right](H)=F\left[\rho_{B, \tau \mid B}\right] \circ F\left[\rho_{V, B}\right](H)=(H \mid B) /(\tau \mid B)$, by functoriality. Therefore $(G / \tau) \mid(\sigma / \tau)$ and $(G \mid \sigma) / \tau$ are identical, and thus isomorphic.

Let $\rho_{V, \sigma}: V \rightarrow \sigma$ and $\rho_{\tau, \sigma / \tau}: \tau \rightarrow \sigma / \tau$ be the canonical surjections, and let $g: \sigma \rightarrow \sigma / \tau$ be the natural bijection. By definition, $H / \sigma=F\left[\rho_{V, \sigma}\right](H)$ and $(H / \tau) /(\sigma / \tau)=F\left[\rho_{\tau, \sigma / \tau}\right] \circ F\left[\rho_{V, \tau}\right](H)$. Therefore $(H / \tau) /(\sigma / \tau)=F[g](H / \sigma)$, since $g \circ \rho_{V, \sigma}=\rho_{\tau, \sigma / \tau} \circ \rho_{V, \tau}$. Hence the third identity follows.

For any $\mathrm{H}$-species $F$, define $\mathcal{H}_{F}$ to be the free module over $K$ with basis consisting of all isomorphism classes of $\left(\exp F_{0}\right)$-structures. The natural product of types of assemblies, equation 3.5, gives $\mathcal{H}_{F}$ an algebra structure, and the correspondence $[G] \leftrightarrow[\{G\}]$ defines an isomorphism between the polynomial algebra $K[\tilde{F}]$ and $\mathcal{H}_{F}$.

Define linear maps $\Delta_{\mathcal{H}}: \mathcal{H}_{F} \rightarrow \mathcal{H}_{F} \otimes \mathcal{H}_{F}$ and $\epsilon: \mathcal{H}_{F} \rightarrow K$ by

$$
\Delta_{\mathcal{H}}[G]=\sum_{\substack{\sigma \in \Pi(V) \\ \sigma \leq \pi_{G}}}[G \mid \sigma] \otimes[G / \sigma]
$$

and

$$
\epsilon[G]= \begin{cases}1 & \text { if } \pi_{G} \text { consists of singletons or is empty } \\ 0 & \text { otherwise }\end{cases}
$$

for any $\left(\exp F_{0}\right)$-structure $G$ on a set $V$.

Proposition 4.2. For any $H$-species $F, \mathcal{H}_{F}$ is a commutative $K$-bialgebra with coproduct $\Delta_{\mathcal{H}}$ and counit $\epsilon$ defined as above.

Proof. The proof that $\Delta_{\mathcal{H}}$ is well-defined by equation 4.1 is similar to the proof that $\Delta$ is well-defined in proposition 2, and is thus omitted. For coassociativity, suppose $G$ is an assembly of $F$-structures on a set $V$. Then

$$
\left(\Delta_{\mathcal{H}} \otimes I\right) \circ \Delta_{\mathcal{H}}[G]=\sum_{\substack{\tau, \sigma \in \Pi^{\prime}(V) \\ \tau \leq \sigma \leq \pi_{G} \\ 13}}[(G \mid \sigma) \mid \tau] \otimes[(G \mid \sigma) / \tau] \otimes[G / \sigma]
$$


While

$$
\begin{aligned}
\left(I \otimes \Delta_{\mathcal{H}}\right) \circ \Delta_{\mathcal{H}}[G] & =\sum_{\substack{\tau \in \Pi(V) \\
\tau \leq \pi_{G}}} \sum_{\substack{\gamma \in \Pi(\tau) \\
\gamma \leq \pi_{G} / \tau}}[G \mid \tau] \otimes[(G / \tau) \mid \gamma] \otimes[(G / \tau) / \gamma] \\
& =\sum_{\substack{\tau, \sigma \in \Pi(V) \\
\tau \leq \sigma \leq \pi_{G}}}[G \mid \tau] \otimes[(G / \tau) \mid(\sigma / \tau)] \otimes[(G / \tau) /(\sigma / \tau)],
\end{aligned}
$$

which is equal to the right-hand side of equation 4.2 , by proposition 4.1 . Thus $\Delta_{\mathcal{H}}$ is coassociative.

To see that $\Delta_{\mathcal{H}}$ is an algebra map, suppose $G_{1}$ and $G_{2}$ are assemblies of $F$-structures on sets $V_{1}$ and $V_{2}$, respectively. Then $\Delta_{\mathcal{H}}\left(\left[G_{1}\right]\left[G_{2}\right]\right)$ is given by

$$
\begin{aligned}
\Delta_{\mathcal{H}}\left[G_{1}+G_{2}\right] & =\sum_{\sigma \leq \pi_{G_{1}} \cup \pi_{G_{2}}}\left[\left(G_{1}+G_{2}\right) \mid \sigma\right] \otimes\left[\left(G_{1}+G_{2}\right) / \sigma\right] \\
& =\sum_{\sigma_{1} \leq \pi_{G_{1}}} \sum_{\sigma_{2} \leq \pi_{G_{2}}}\left[G_{1} \mid \sigma_{1}\right]\left[G_{2} \mid \sigma_{2}\right] \otimes\left[G_{1} / \sigma_{1}\right]\left[G_{2} / \sigma_{2}\right] \\
& =\Delta_{\mathcal{H}}\left[G_{1}\right] \Delta_{\mathcal{H}}\left[G_{2}\right] .
\end{aligned}
$$

Hence $\Delta_{\mathcal{H}}$ is an algebra map. It is easy to see that $\epsilon$ is also an algebra map. Thus $\mathcal{H}_{F}$ is a bialgebra.

If $\alpha: F \rightarrow E$ is a morphism of $\mathrm{H}$-species, then there is a corresponding algebra map $\tilde{\alpha}: \mathcal{H}_{F} \rightarrow \mathcal{H}_{E}$ defined by $\tilde{\alpha}[\{G\}]=\left[\left\{\alpha_{V}(G)\right\}\right]$, whenever $G$ is an $F$-structure on a non-empty set $V$.

Proposition 4.3. If $\alpha: F \rightarrow E$ is a morphism of $H$-species, then the corresponding map $\tilde{\alpha}: \mathcal{H}_{F} \rightarrow \mathcal{H}_{E}$ is a bialgebra map.

Proof. By definition, $\tilde{\alpha}$ is an algebra map. Let $G$ be an $F$-structure on a non-empty set $V$. Then

$$
\Delta_{\mathcal{H}} \circ \tilde{\alpha}[\{G\}]=\sum_{\sigma \in \Pi(V)}\left(\prod_{B \in \sigma}\left[\left\{\alpha_{V}(G) \mid B\right\}\right]\right) \otimes\left[\left\{\alpha_{V}(G) / \sigma\right\}\right] .
$$

Since $\alpha$ is a natural transformation, this can be written as

$$
\sum_{\sigma \in \Pi(V)}\left(\prod_{B \in \sigma}\left[\left\{\alpha_{B}(G \mid B)\right\}\right]\right) \otimes\left[\left\{\alpha_{\sigma}(G / \sigma)\right\}\right]
$$

which is equal to $(\tilde{\alpha} \otimes \tilde{\alpha}) \circ \Delta_{\mathcal{H}}[\{G\}]$. Also, it is clear that $\epsilon \circ \tilde{\alpha}=\epsilon$, hence $\tilde{\alpha}$ is a bialgebra map.

Thus we have the following theorem.

Theorem 4.4. The correspondence $F \mapsto \mathcal{H}_{F}$ is a functor from the category of $H$-species and natural transformations to the category of commutative bialgebras and bialgebra maps. 
4.3. Hopf Algebras of Simple H-Species. A species $F$ is simple, if $|F(V)|=1$ whenever $|V|=1$. If $F$ is any simple species, then the exponential $E=\exp F_{0}$ is an $\mathbf{I}$-species. For if $\alpha: U \rightarrow V$ is an injection and $G$ is an assembly of $F$-structures on $U$, then the assembly $E[\alpha](G)$ on $V$, called the extension of $G$ to $V$, can be defined by

$$
E[\alpha](G)=E[\bar{\alpha}](G)+\sum_{x \in V-R(\alpha)}\{F(\{x\})\},
$$

where $\bar{\alpha}$ is the bijection defined by $\alpha$ from $U$ onto the range $R(\alpha)$ of $\alpha$, and $\{F(\{x\})\}$ is the assembly consisting of the unique $F$-structure on the set $\{x\}$.

Let $F$ be a simple H-species. Then $\exp F$ is an $\mathbf{I}_{p}$-species, where assemblies of $F$-structures restrict as usual by equation 3.3 and extensions are given as above. It follows that two assembies of F-structures are weakly isomorphic if and only if they are they are isomorphic after deleting all of their singleton components.

Let $\hat{\mathcal{H}}_{F}$ be the free $K$-module having the set of all weak isomorphism classes of non-empty (exp $\left.F_{0}\right)$-structures as a basis. Defining products of weak isomorphism classes by

$$
\langle G\rangle\langle H\rangle=\langle G+H\rangle
$$

makes $\hat{\mathcal{H}}_{F}$ an algebra, isomorphic to the polynomial algebra over $K$ having types of $F$-structures on sets of two or more elements as indeterminates. If $|V|=1$ and $G$ is the assembly consisting of the unique $F$-structure on $V$, then $\langle G\rangle$ is the multiplicative identity of $\hat{\mathcal{H}}_{F}$.

Define linear maps $\Delta_{\mathcal{H}}: \hat{\mathcal{H}}_{F} \rightarrow \hat{\mathcal{H}}_{F} \otimes \hat{\mathcal{H}}_{F}$ and $\epsilon: \hat{\mathcal{H}}_{F} \rightarrow K$ by

$$
\Delta_{\mathcal{H}}\langle G\rangle=\sum_{\substack{\sigma \in \Pi(V) \\ \sigma \leq \pi_{G}}}\langle G \mid \sigma\rangle \otimes\langle G / \sigma\rangle
$$

and

$$
\epsilon\langle G\rangle= \begin{cases}1 & \text { if } \pi_{G} \text { consists of singletons } \\ 0 & \text { otherwise }\end{cases}
$$

for any non-empty $\left(\exp F_{0}\right)$-structure $G$ on a set $V$.

Proposition 4.5. For any simple H-species $F, \hat{\mathcal{H}}_{F}$ is a commutative Hopf algebra over $K$ with $\Delta_{\mathcal{H}}$ and $\epsilon$ defined as above. The antipode $S: \hat{\mathcal{H}}_{F} \rightarrow \hat{\mathcal{H}}_{F}$ is given by

$$
S\langle G\rangle=\sum_{k=0}^{|V|} \sum(-1)^{k}\left\langle\left(G \mid \sigma_{1}\right) / \sigma_{0}\right\rangle\left\langle\left(G \mid \sigma_{2}\right) / \sigma_{1}\right\rangle \cdots\left\langle\left(G \mid \sigma_{k}\right) / \sigma_{k-1}\right\rangle,
$$

where the inner sum is over all chains $\sigma_{0}<\sigma_{1} \cdots<\sigma_{k}$ in $\Pi(V)$ having $\sigma_{k}=\pi_{G}$ and $\sigma_{0}$ the partition of $V$ into singletons. 
The proof that $S$ is an antipode is essentially the same as that given in proposition 3.4. Both antipode formulas are special cases of the general formula for antipodes of incidence Hopf algebras given in [12].

The following theorem is now apparent.

Theorem 4.6. The correspondence $F \mapsto \hat{\mathcal{H}}_{F}$ is a functor from the category of simple H-species to the category of commutative Hopf algebras.

Example 4.2 (The Faà di Bruno Hopf Algebra). The uniform species $U$ is a simple $\mathrm{H}$-species. The corresponding Hopf algebra $\hat{\mathcal{H}}_{U}$ is isomorphic to the Faà di Bruno Hopf algebra, investigated in [4], [12] and [3]. In this case, the antipode formula 4.4 has been shown (in [3]) to be equivalent to the Lagrange formula for the inverse of a formal power series under composition.

4.4. Comodule Coalgebras of $\mathbf{H}$-Species. Suppose $\mathcal{B}_{F}$ and $\hat{\mathcal{H}}_{F}$ are, respectively, the cocommutative and non-cocommutative Hopf Algebras corresponding to a simple H-species $F$. Define a linear map $\psi: \mathcal{B}_{F} \rightarrow \hat{\mathcal{H}}_{F} \otimes \mathcal{B}_{F}$ by $\psi(1)=1 \otimes 1$ and

$$
\psi[G]=\sum_{\sigma \in \Pi(V)}\langle G \mid \sigma\rangle \otimes[G / \sigma],
$$

whenever $G$ is an $F$-structure on a set $V$, where $|V| \geq 1$.

Proposition 4.7. The pair $\left(\mathcal{B}_{F}, \psi\right)$ is a left $\hat{\mathcal{H}}_{F}$-comodule coalgebra.

Proof. To see that $\mathcal{B}_{F}$ is a left $\hat{\mathcal{H}}_{F}$-comodule, one must show that $(\epsilon \otimes I) \circ \psi=$ $I$ and $\left(\Delta_{\mathcal{H}} \otimes I\right) \circ \psi=(I \otimes \psi) \circ \psi$, where $I$ denotes either the identity map on $\mathcal{B}_{F}$ or that on $\hat{\mathcal{H}}_{F}$, depending on where it appears in an expression. The first of these equations is trivial. The proof of the second is essentially identical to the verification that $\Delta_{\mathcal{H}}$ is coassociative.

The statement that $\mathcal{B}_{F}$ is a left $\hat{\mathcal{H}}_{F}$-comodule coalgebra (see [1], p. 137), means that the structure maps $\Delta$ and $\epsilon$ of $\mathcal{B}_{F}$ are $\hat{\mathcal{H}}_{F}$-colinear. In other words,

$$
(I \otimes \Delta) \circ \psi=(\mu \otimes I \otimes I) \circ(I \otimes T \otimes I) \circ(\psi \otimes \psi) \circ \Delta
$$

and

$$
(I \otimes \epsilon) \circ \psi=(\eta \otimes I) \circ \epsilon,
$$

where $\mu: \hat{\mathcal{H}}_{F} \otimes \hat{\mathcal{H}}_{F} \rightarrow \hat{\mathcal{H}}_{F}$ and $\eta: K \rightarrow \hat{\mathcal{H}}_{F}$ are the multiplication and unit of $\hat{\mathcal{H}}_{F}$, and $T: \mathcal{B}_{F} \otimes \hat{\mathcal{H}}_{F} \rightarrow \hat{\mathcal{H}}_{F} \otimes \mathcal{B}_{F}$ is the twist map, determined by $T([G] \otimes\langle H\rangle)=\langle H\rangle \otimes[G]$, for all $[G] \otimes\langle H\rangle \in \mathcal{B}_{F} \otimes \hat{\mathcal{H}}_{F}$.

Equation 4.6 is trivial. In order to verify equation 4.5, suppose $G \in F(V)$, where $|V| \geq 1$. Then $(I \otimes \Delta) \circ \psi[G]$ is given by

$$
\sum_{\sigma \in \Pi(V)} \sum_{\gamma \subseteq \sigma}\langle G \mid \sigma\rangle \otimes[(G / \sigma) \mid \gamma] \otimes[(G / \sigma) \mid(\sigma-\gamma)]
$$


On the other hand, $(\psi \otimes \psi) \circ \Delta[G]$ is given by

$$
\sum_{U \subseteq V} \sum_{\sigma_{1} \in \Pi(U)} \sum_{\sigma_{2} \in \Pi(V-U)}\left\langle(G \mid U) \mid \sigma_{1}\right\rangle \otimes\left[(G \mid U) / \sigma_{1}\right] \otimes\left\langle(G \mid(V-U)) \mid \sigma_{2}\right\rangle \otimes\left[(G \mid(V-U)) / \sigma_{2}\right] .
$$

Hence, $(\mu \otimes I \otimes I) \circ(I \otimes T \otimes I) \circ(\psi \otimes \psi) \circ \Delta[G]$ equals

$$
\sum_{U \subseteq V} \sum_{\sigma_{1} \in \Pi(U)} \sum_{\sigma_{2} \in \Pi(V-U)}\left\langle G \mid\left(\sigma_{1} \cup \sigma_{2}\right)\right\rangle \otimes\left[(G \mid U) / \sigma_{1}\right] \otimes\left[(G \mid(V-U)) / \sigma_{2}\right],
$$

which is equal to (4.7), by functoriality. Thus equation 4.5 follows.

Suppose $\mathcal{H}_{F}$ is the bialgebra of the H-species $F$ and $\hat{\psi}: \mathcal{B}_{F} \rightarrow \mathcal{H}_{F} \otimes \mathcal{B}_{F}$ is defined by $\hat{\psi}(1)=1 \otimes 1$ and

$$
\hat{\psi}[G]=\sum_{\sigma \in \Pi(V)}[G \mid \sigma] \otimes[G / \sigma],
$$

whenever $G$ is an $F$-structure on a set $V$, where $|V| \geq 1$. A proof identical to that of proposition 4.7, with square brackets replacing all angle brackets, shows that the pair $\left(\mathcal{B}_{F}, \hat{\psi}\right)$ is a left $\mathcal{H}_{F}$-comodule coalgebra.

\section{INVARIANTS OF STRUCTURES}

If $\mathcal{H}$ is any Hopf algebra over $K$ then the subset $\operatorname{Alg}(\mathcal{H}, K)$ of the dual algebra $\mathcal{H}^{*}$, consisting of all algebra maps from $\mathcal{H}$ to the ring $K$, forms a group under product in $\mathcal{H}^{*}$, with the counit $\epsilon$ as an identity. The inverse of a map $f \in \operatorname{Alg}(\mathcal{H}, K)$ is given by $f^{-1}=f \circ S$, where $S$ is the antipode of $\mathcal{H}$ (see [13] for details).

Let $F$ be a simple $\mathrm{H}$-species, and let $\hat{\mathcal{H}}_{F}$ be the corresponding Hopf algebra. The product of elements $f$ and $g$ in $\hat{\mathcal{H}}_{F}^{*}$ is given by

$$
f \cdot g\langle G\rangle=\sum_{\substack{\sigma \in \Pi(V) \\ \sigma \leq \pi_{G}}} f\langle G \mid \sigma\rangle g\langle G / \sigma\rangle,
$$

for any assembly of $F$-structures $G$ on a set $V$ having underlying partition $\pi_{G}$.

The dual $\hat{\mathcal{H}}_{F}^{*}$ is called the algebra of invariants of $F$-structures. The group of algebra maps $\operatorname{Alg}\left(\hat{\mathcal{H}}_{F}, K\right)$ is denoted by $\mathcal{M}_{F}$ and called the group of $m u l$ tiplicative invariants of $F$-structures. Any $f \in \mathcal{M}_{F}$ is uniquely determined by the values it takes on weak-isomorphism classes of $F$-structures.

Since $\mathcal{B}_{F}$ is a left $\hat{\mathcal{H}}_{F}$-comodule, it follows that $\mathcal{B}_{F}$ is a left $\hat{\mathcal{H}}_{F}^{*}$-module, where the action of $f \in \hat{\mathcal{H}}_{F}^{*}$ on $[G] \in \mathcal{B}_{F}$ is given by

$$
f[G]=\sum_{\pi \in \Pi(V)} f^{-1}\langle G \mid \pi\rangle[G / \pi],
$$

for $G \in F(V)$ and $V \neq \emptyset$. If $V=\emptyset$, then $[G]=1$, and $f \cdot 1=f(1) \cdot 1$. 
For any $f \in \mathcal{M}_{F}$, let $\bar{f}: \mathcal{B}_{F} \rightarrow \mathcal{B}_{F}$ be the left multiplication map $[G] \rightarrow$ $f[G]$. It is a formal consequence of proposition 4.7 that $\bar{f}$ is a coalgebra automorphism of $\mathcal{B}_{F}$. Thus we have the following proposition.

Proposition 5.1. For any simple $H$-species $F$, the correspondence $f \rightarrow$ $\bar{f}$ defines an action of the group of multiplicative invariants $\mathcal{M}_{F}$ on the coalgebra $\mathcal{B}_{F}$.

Example 5.1 (Simple Graphs). Let $G_{s}$ be the simple H-species of simple graphs, and let $K$ be the ring of integers. Define multiplicative invariants $\nu, \eta, \zeta \in \mathcal{M}_{G_{s}}$ by

$$
\begin{aligned}
& \nu\langle G\rangle= \begin{cases}1 & \text { if } G \text { is connected } \\
0 & \text { otherwise, }\end{cases} \\
& \eta\langle G\rangle= \begin{cases}1 & \text { if } G \text { has no edges } \\
0 & \text { otherwise, }\end{cases}
\end{aligned}
$$

and

$$
\zeta\langle G\rangle=1
$$

for all graphs $G$. The inverse $\mu$ of $\zeta$ in $\mathcal{M}_{G_{s}}$ is given by $\mu\langle G\rangle=(-1)^{n-1}(n-$ $1)$ !, whenever $G$ has $n$ vertices. The invariant $\mu$ is the Möbius function of the lattice of partitions (see [11]).

The product $\nu \cdot \eta$ is given by

$$
\nu \cdot \eta\langle G\rangle=\sum_{\sigma \in \Pi(V)} \nu\langle G \mid \sigma\rangle \eta\langle G / \sigma\rangle,
$$

for any graph $G$ with vertex-set $V$. The single non-vanishing term of this sum occurs when $\sigma$ is equal to $\pi_{G}$, the partition of $G$ into connected components. Therefore, we have the identity $\nu \cdot \eta=\zeta$, or equivalently, $\eta^{-1}=\mu \cdot \nu$ in $\mathcal{M}_{G_{s}}$.

The automorphism of $\mathcal{B}_{G_{s}}$ corresponding to $\eta^{-1}$ is thus given by

$$
\bar{\mu} \circ \bar{\nu}[G]=\sum[G / \sigma]
$$

where the sum is over all color partitions $\sigma$ (i.e. partitions of the vertex set into independent sets) of $G$. The automorphism $\bar{\mu} \circ \bar{\nu}$ of $\mathcal{B}_{G_{s}}$ thus generalizes the chromatic polynomial of a graph.

\section{REFERENCES}

[1] E. Abe, Hopf Algebras (Cambridge University Press, Cambridge, 1980).

[2] H. Décoste, G. LaBelle and P. Leroux, Une Approche Combinatoire Pour L'itération de Newton-Raphson, Advances in Applied Mathematics 3 (1982), 407-416.

[3] M. Haiman and W. Schmitt, Antipodes, Incidence Coalgebras and Lagrange Inversion in One and Several Variables, Journal of Combinatorial Theory A 50 (1989), 172-185.

[4] S. Joni and G.-C. Rota, Coalgebras and Bialgebras in Combinatorics, Studies in Applied Mathematics 61 (1979), 93-139.

[5] A. Joyal, Une Théorie Combinatoire des Séries Formelles, Advances in Mathematics 42 (1981), 1-82.

[6] G. Labelle, Une Nouvelle Démonstration Combinatoire des Formules d'Inversion de Lagrange, Advances in Mathematics 42 (1981), 217-247. 
[7] G. Labelle, Éclosions Combinatoires Appliquées à l'Inversion Multidimensionelle des Séries Formelles, Journal of Combinatorial Theory A 39 (1985), 52-82.

[8] G. Labelle, Une Combinatoire Sous-Jacente au Théorème des Fonctions Implicites, Journal of Combinatorial Theory A 40 (1985), 377-393.

[9] G. Labelle, On Combinatorial Differential Equations, Journal of Mathematical Analysis and Applications 113 (1986), 344-381.

[10] J. Labelle, Applications Diverses de la Théorie Combinatoire des Espèces de Structures, Ann. Sci. Math. Québec 7 (1983), 58-94.

[11] G. C. Rota, On the Foundations of Combinatorial Theory I, Theory of Mobius Functions, Z. Wahrscheinlichkeitstheorie 2 (1964), 340-368.

[12] W. Schmitt, Antipodes and Incidence Coalgebras, Journal of Combinatorial Theory A 46 (1987), 264-290.

[13] M. Sweedler, Hopf Algebras (Benjamin, New York, 1969). 